\title{
An Optimal On-Off Controller with Switching Costs using Non-linear Binary Programming
}

\author{
Biju Edamana and Kenn Oldham
}

\begin{abstract}
Autonomous systems based on MEMS devices may often be provided with very limited computational and power capacity, if control circuitry and power sources are to be miniaturized along with the electromechanical components. OnOff control can serve as an efficient methods of regulating motion of MEMS structures when power is extremely limited by allowing control to be performed using simple driving circuits and few transitions between 'on'- and 'off'-states. In particular, this is highly desirable for micro-robotics applications based on piezoelectric actuation. In this paper a binary programming method is used to optimize a cost function that consists of the number of switching transitions and on-time for a lineardiscrete system, as the system is steered to a desired final state. This can be used to minimize power consumption in piezoelectric actuators as they move a micro-robotic leg joint to a desired position. A set of test cases is examined to explore behavior of the optimization procedure.
\end{abstract}

\section{INTRODUCTION}

Autonomous operation of micro-electromechanical systems (MEMS) requires strict attention to power consumption during servo control, so that MEMS components can be effectively operated with miniature power sources. Many MEMS devices rely on piezoelectric or electrostatic actuators to produce motion, where power consumption is primarily related to a capacitive load. In these situations, On-Off control can be an important method for performing servo control within a limited power budget, because switching On-Off drive circuits have lower energy losses than ana$\log$ amplifiers, and reducing switching frequency relative to pulse-width-modulation (PWM) can further reduce the energy consumption [1] [2].

The goal of this work is to minimize energy loss while driving a system to a desired states with an On-Off controller when significant energy losses are incurred to switch between 'on' an 'off' inputs. This problem is inspired by the need for a controller to produce efficient motions of a microrobotic leg joint driven by piezoelectric actuators, such as the prototype joint and actuator shown in Fig. 1. In this application, energy is lost when the actuator is charged or discharged as well as through leakage resistances in the actuator or driving circuit when the controller input is 'on.' As practical position sensors are not yet available for the prototype structures, control would be applied in open-loop, using sequences of 'on' and 'off' inputs predetermined using the algorithms described in this paper.

B. Edamana and K. Oldham are with the Department of Mechanical Engineering, University of Michigan, Ann Arbor, MI 48109, USA bi j, oldhameumich.edu

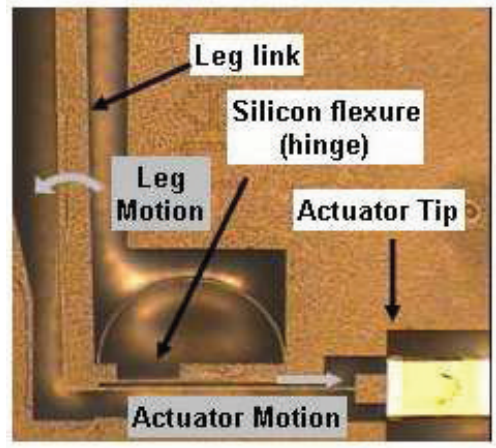

Fig. 1. Prototype micro-robotic leg link

Previous researchers have developed methods for minimizing 'on'-time of an On-Off controller while achieving certain goals [3], or minimizing total time required to complete a task using On-Off control [4], but these methods did not incorporate switching costs. In this paper, a cost function consisting of both on-time and number of switches is considered. Capacitive costs associated with switching result in a nonlinearity in the cost function, increasing solution difficulty. In response, a method for converting this cost function to a quadratic form is given. Once in this form, there are many solution methods to minimize the cost function, of which two main types have been studied. In the first type of solution method, the switched system is considered as a hybrid system and a hybrid solver is used (a combination of logic solver such a satisfiability solver (SAT) or constraint programming $(\mathrm{CP})$ plus an integer programming solver) [5][6]. The second type of solution method uses binary programming to optimize a penalty function which is a weighted sum of constraints and the objective function [7]. The second method is adopted for this procedure due to its simplicity in producing a convex optimization problem.

The paper is arranged as follows. First, a description of the system is given. Then, a detailed derivation of an optimal On-Off controller is given using a non-linear binary programming technique. A summary of results when applied to the model of a micro-robotic leg joint follows. Finally, key conclusions are summarized.

\section{SYSTEM AND PROBLEM DESCRIPTION}

As a demonstration of this approach, a lumped-parameter model of the prototype system is analyzed. This prototype, as shown in Fig. 1 and schematically in Fig. 2, consists of a rigid micro-robotic leg rotating about an elastic flexure, with the silicon flexure modeled as a rotary spring, 
TABLE I

NOMINAL PARAMETER VALUES FOR ON-OFF CONTROLLER TEST SCENARIOS

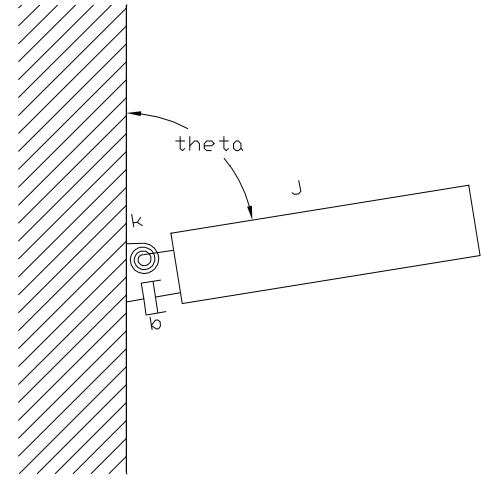

Fig. 2. Single mass system

$k$, and a representative damping coefficient obtained from experimental tests. These parameters are essentially linear over the range of motions that can be produced by the piezoelectric actuators. The system is represented by the following differential equations, with nominal values for the parameters given in Table I.

$$
J \ddot{\theta}+b \dot{\theta}+k \theta=G u(t)
$$

where, $\theta$ is the angle of rotation, $u$ is the input voltage, $J$ is the rotational inertia of the leg, $b$ is the damping coefficient, $k$ is the spring constant, and $G$ is the actuator gain.

The system can be represented in state space format with state vector $x=\left[\begin{array}{l}x_{1} \\ x_{2}\end{array}\right]$ where: $\mathrm{x}_{1}=$ angle of rotation, $\theta$, $\mathrm{x}_{2}=$ angular velocity, $\dot{\theta}$.

$$
\begin{aligned}
{\left[\begin{array}{c}
\dot{x_{1}} \\
\dot{x_{2}}
\end{array}\right] } & =\left[\begin{array}{cc}
0 & 1 \\
-k / J & -b / J
\end{array}\right]\left[\begin{array}{l}
x_{1} \\
x_{2}
\end{array}\right]+\left[\begin{array}{c}
0 \\
G / J
\end{array}\right] \\
y & =\left[\begin{array}{ll}
1 & 0
\end{array}\right] x
\end{aligned}
$$

The system can be discretized in terms of index $k$ to standard discrete state-space form

$$
\begin{aligned}
x\left((k+1) T_{s}\right) & =A_{d} x(k T s)+B_{d} u\left(k T_{s}\right) \\
y\left(k T_{s}\right) & =\left[\begin{array}{ll}
1 & 0
\end{array}\right] x\left(k T_{s}\right)
\end{aligned}
$$

where $A_{d}$ and $B_{d}$ are state and input matrices. For the sample problem, these matrices are formed by continuous to discrete conversion of the system in 2 using a zero order hold with sampling time $T_{s}$.

Under On-Off control, the input $u(k T)$ is limited to to one of two levels, zero or $U_{\max }$. Inputs to the system can thus be rewritten in terms of binary inputs $u_{1,2, \ldots, n}$ where

$$
\begin{array}{r}
u\left(k T_{s}\right)=U_{\max } u_{k} \\
u_{k} \in(0,1)
\end{array}
$$

\begin{tabular}{|l|l|}
\hline Parameters & Value \\
\hline$J\left(\right.$ kg.m $\left.{ }^{2}\right)$ & $1.4 * 10^{-12}$ \\
$b(\mathrm{~N} . \mathrm{m} . \mathrm{s} / \mathrm{rad})$ & $3.4 * 10^{-11}$ \\
$k(\mathrm{~N} . \mathrm{m} / \mathrm{rad})$ & $3.2 * 10-6$ \\
$G(\mathrm{~N} . \mathrm{m} / \mathrm{V})$ & $8 * 10-8$ \\
$R(\Omega)$ & $3 * 10^{9}$ \\
$C(\mathrm{~F})$ & $1 * 10^{-9}$ \\
$U_{\max }(\mathrm{V})$ & 40 \\
$T_{s}(\mathrm{~s})$ & 0.001 \\
\hline
\end{tabular}

which simplifies the problem to a binary programming problem and it can be converted to a convex optimization problem comparatively easily.

\section{OPtIMAL ON-OFF CONTROLLER}

\section{A. Objective}

The problem here is to attain a desired final state $x_{d}$ at a desired final time $t_{f}=n T_{s}$ over $n$ time steps using minimum energy. This will be done by minimizing a cost function that includes energy use by the actuator while constraining the final states to be at or near their desired values. Moreover, there is the additional constraint in the inputs that $u \in\{0,1\}$. The effect of these constraints is that there are only a finite reachable points for the system, as compared to the entire $R^{2}$ case when arbitrary input levels can be applied. These additional constraints can be shown pictorially as in Fig. 3. This figure shows the all possible $2^{16}$ points reachable by the system at the end of 16 time steps, starting from the origin. Visual representation in this manner can aid in identifying desired final points that the system has a reasonable possibility of reaching with limited error.

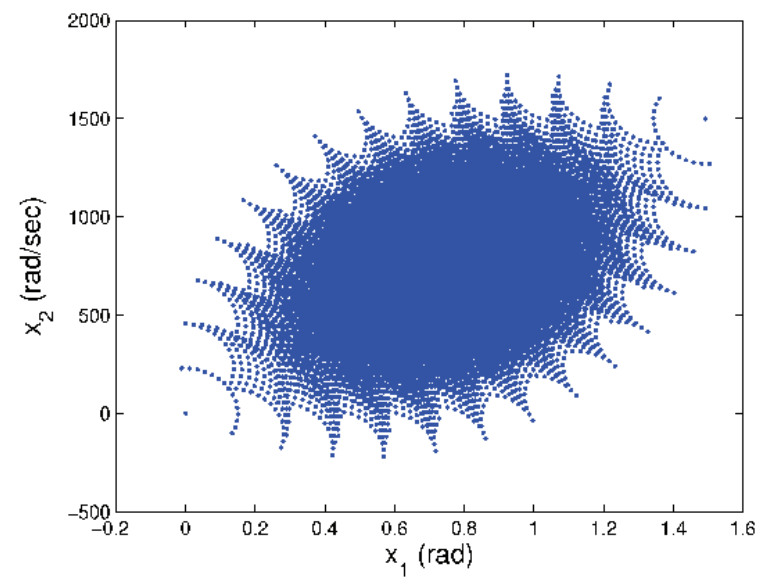

Fig. 3. All possible states after 16 time steps

\section{B. Solution overview}

Given a set of desired final states, the problem becomes a non-linear binary programming problem with $m$ constraints, where $m$ denotes the number of states, which is equal 
to 2 in this case. In our solution procedure we will first convert this problem to an unconstrained non-linear binary programming problem, then to a constrained non-linear quadratic programming problem with $n$ block constraints, which is a continuous convex minimization problem and hence comparatively easy to solve.

\section{Step 1 - Problem statement}

The objective function consists of two parts, $J_{C}$ and $J_{R}$, corresponding to capacitive and resistive energy losses in the system, respectively. A piezoelectric actuator acts as a capacitor when voltage is applied, and the first part of the objective function includes energy lost from the system during charging of the actuator capacitor and when the charged actuator is discharged. Mathematically, when 'on'state magnitude $U_{\max }$ is taken to be the 'on' voltage applied to the actuators, this energy is expressed as

$$
J_{C}=\sum_{k=0}^{n} \frac{1}{2} C U_{\max }^{2}\left(u_{k}-u_{k-1}\right)^{2}
$$

where $C$ is the capacitance of the piezoelectric actuator. In more a more general system, the quantity $C U_{\max }^{2} / 2$ could be replaced by an arbitrary "cost-to-switch".

The second part of the objective function includes energy lost to resistive dissipation due to leakage in the On-Off drive circuit or through the piezoelectric actuator,

$$
J_{R}=\sum_{k=0}^{n} \frac{U_{\max }^{2}}{R} T_{s} u_{k}
$$

where $R$ is the resistance of the system. Again, in a more eneral case of On-Off control, the quantity $U_{\max }^{2} / R$ could be replaced with an arbitrary "cost-to-hold" in the 'on' position.

The total objective function is converted to quadratic form by noting that total cost $J=J_{C}+J_{R}$ can be manipulated as follows:

$$
\begin{aligned}
J= & \min \left\{\left\{U_{\max }^{2} / 2 * C *\left\{u_{1}^{2}+\left(u_{1}-u_{2}\right)^{2}\right.\right.\right. \\
& \left.+\ldots+\left(u_{n-1}-u_{n}\right)^{2}\right\} \\
+ & \left.U_{\max }^{2} / R * T s *\left\{u_{1}+u_{2}+\ldots+u_{n}\right\}\right\} \\
= & \min \left\{U_{\max }^{2} / 2 * C *\left\{2 u_{1}^{2}-2 u_{1} u_{2}+2 u_{2}^{2}+\right.\right. \\
& \left.\quad \ldots+2 u_{n-1}^{2}-2 u_{n-1} u_{n}+u_{n}^{2}\right\} \\
& \left.+U_{\max }^{2} / R * T s *\left\{u_{1}+u_{2}+\ldots+u_{n}\right\}\right\} \\
= & \min \left\{U_{\max }^{2} / 2 * C * \bar{u}^{T} Q_{1} \bar{u}+\right. \\
& \left.U_{\max }^{2} / R * T s * Q_{2} \bar{u}\right\}
\end{aligned}
$$

where,

$$
\begin{aligned}
Q 1 & =\left[\begin{array}{cccc}
2 & -1 & \cdot & 0 \\
-1 & 2 & \cdot & 0 \\
0 & \cdot & \cdot & \cdot \\
0 & \cdot & 2 & -1 \\
0 & \cdot & -1 & 1
\end{array}\right] \\
Q 2 & =\left[\begin{array}{lllll}
1 & 1 & \cdot & \cdot & 1
\end{array}\right]
\end{aligned}
$$

$n$ binary constraints are fixed by the On-Off nature of the system, and are denoted by $c_{i}^{\prime} \mathrm{s}$, with $i=$ to $n$,

$$
u_{i} \in\{0,1\}
$$

An additional $m$ constraints are fixed by the desired final states. For the two-state prototype system considered here, these constraints are denoted as $c_{n+1}$ and $c_{n+2}$ and given by the dynamics of the system as

$$
x_{f}(n)=\sum_{j=0}^{n-1} A_{d}^{n-j-1} B_{d} u(j)+A^{n} x(0)
$$

where, $x(0)$ is assumed to be zero without any lose of generality.

\section{Step 2 - Conversion to an unconstrained binary non- linear programming problem}

The constraints $c_{n+1}$ and $c_{n+2}$ are added to objective function using weights $\mu_{1}$ and $\mu_{2}$ which makes the problem a binary unconstrained problem, as the binary $c_{i}$ 's remain present. This modifies the objective function to the following form,

$$
J_{1}=\min \left\{J+\mu_{1} *\left(c_{n+1}\right)^{2}+\mu_{2} *\left(c_{n+2}\right)^{2}\right\}
$$

subject to the $n$ binary constraints

\section{E. Step 3 - Conversion to a continuous convex minimization problem}

Consider the following two optimization problems $(B Q)$ and $(\overline{B Q})$,

$$
\begin{array}{ll}
(B Q) \quad & \min _{\zeta \in\{-1,1\}^{n}} q(\zeta)=1 / 2 \zeta^{T} Q \zeta+b^{T} \zeta \\
(\overline{B Q}) \quad & \min q(\zeta)=1 / 2 \zeta^{T} Q \zeta+b^{T} \zeta \\
& \text { s.t. } \zeta_{i}^{2} \leq 1, i=1, \ldots, n .
\end{array}
$$

Problem $\overline{B Q}$ is a convex continuous optimization problem which gives real numbers as solution; moreover, it is comparatively easy to solve. From [7], the solution of $B Q$ can be obtained by rounding off the real solution of $\overline{B Q}$ to $\{-1,1\}$.

Thus, if the problem given in equation (12) is converted to the above form, it is comparatively easy to solve. Since the problem in (12) has the input variables $u \in\{0,1\}$, they must first be changed to a new set of variables $v \in\{-1,-1\}$ by the coordinate transformation $v_{i}=2 u_{i}-1$. In vector form, we can perform this transformation using vector $\bar{e}=$ $\left[\begin{array}{ccccc}1 & 1 & \cdot & \cdot & 1\end{array}\right]^{T}$. Grouping $u_{i}$ 's and $v_{i}$ 's into vectors $\bar{u}$ and $\bar{v}$, we could alternately perform the transformation using the equation

$$
\Rightarrow \bar{v}=(\bar{u}+\bar{e}) / 2
$$

The transformation (15) is applied to the objective function and the constraints as follows. The constraints $c_{n+1}$ and $c_{n+2}$ 
can be written as,

$$
\begin{gathered}
c_{n+1}: \sum_{j=0}^{j=n-1} a_{1 j} v_{j}+x_{f 1}=0 \\
c_{n+2}: \sum_{j=0}^{j=n-1} a_{2 j} v_{j}+x_{f 2}=0
\end{gathered}
$$

where

$$
\begin{aligned}
x_{f 1} & =\sum_{j=0}^{n-1}\left(a_{1 j}\right)-2 x_{d}(1) \\
x_{f 2} & =\sum_{j=0}^{n-1}\left(a_{2 j}\right)-2 x_{d}(2)
\end{aligned}
$$

and coefficients $a_{i j}$ are taken from (11)

$$
A_{d}^{n-j-1} B_{d}=\left[\begin{array}{lllll}
a_{11} & a_{12} & \cdot & \cdot & a_{1 n} \\
a_{21} & a_{22} & \cdot & \cdot & a_{2 n}
\end{array}\right]
$$

with $m=2$ in this example.

Now, $\left(c_{n+1}\right)^{2}$ and $\left(c_{n+2}\right)^{2}$ can be written as given below by eliminating the constant terms,

$$
\begin{aligned}
\left(c_{n+1}\right)^{2}= & \left(\sum_{j=0}^{j=n-1} a_{i} v_{i}+x_{f 1}\right)^{2} \\
= & \sum_{j=0}^{j=n-1}\left(a_{i} v_{i}\right)^{2}+2 x_{f 1} \sum_{j=0}^{j=n-1} v_{i} \\
& +\sum_{j=0} \sum_{i=0, i \neq j}^{i=n-1} 2 a_{i} a_{j} v_{i} v_{j} \\
\left(c_{n+2}\right)^{2}= & \left(\sum_{j=0}^{j=n-1} b_{i} v_{i}+x_{f 2}\right)^{2} \\
= & \sum_{j=0}^{j=n-1}\left(b_{i} v_{i}\right)^{2}+2 x_{f 2} \sum_{j=n-1}^{j=n-1} v_{i} \\
& +\sum_{j=0}^{i=n-1} \sum_{i=0, i \neq j} 2 b_{i} b_{j} v_{i} v_{j}
\end{aligned}
$$

This quantity can be written in turn in a matrix form as

$$
\begin{aligned}
& \left(c_{n+1}\right)^{2}=\bar{v}^{T} A_{c_{n+1}} \bar{v}+B_{c_{n+1}} \bar{v} \\
& \left(c_{n+2}\right)^{2}=\bar{v}^{T} A_{c_{n+2}} \bar{v}+B_{c_{n+2}} \bar{v}
\end{aligned}
$$

where,

$$
\begin{aligned}
& A_{c_{n+1}}=\left[\begin{array}{cccc}
a_{1}^{2} & a_{1} a_{2} & \cdot & a_{1} a_{n} \\
a_{1} a_{2} & a_{2}^{2} & \cdot & a_{2} a_{n} \\
\cdot & \cdot & \cdot & \cdot \\
\cdot & \cdot & \cdot & \cdot \\
a_{1} a_{n} & \cdot & \cdot & a_{n}^{2}
\end{array}\right] \\
& A_{c_{n+2}}=\left[\begin{array}{cccc}
b_{1}^{2} & b_{1} b_{2} & \cdot & b_{1} b_{n} \\
b_{1} b_{2} & b_{2}^{2} & \cdot & b_{2} b_{n} \\
\cdot & \cdot & \cdot & \cdot \\
\cdot & \cdot & \cdot & \cdot \\
b_{1} b_{n} & \cdot & \cdot & b_{n}^{2}
\end{array}\right] \\
& B_{c_{n+1}}=2 x_{f 1}\left[\begin{array}{lllll}
a_{1} & a_{2} & \cdot & a_{n}
\end{array}\right] \\
& B_{c_{n+2}}=2 x_{f 2}\left[\begin{array}{lllll}
b_{1} & b_{2} & \cdot & b_{n}
\end{array}\right]
\end{aligned}
$$

Now consider the transformation of the objective function,

$$
\begin{aligned}
u^{T} Q_{1} u+Q_{2} u= & 1 / 2(v+\bar{e})^{T} Q_{1} 1 / 2(v+\bar{e})+Q_{2}(v+\bar{e}) / 2 \\
= & 1 / 4\left(v^{T} Q_{1} v+\bar{e}^{T} Q_{1} v+v^{T} Q_{1} \bar{e}+\right. \\
& \quad \text { constants })+Q_{2} v / 2 \\
= & 1 / 4\left(v^{T} Q_{1} v+2 v(1)\right)+Q_{2} v / 2
\end{aligned}
$$

Thus, the problem can be written in the new coordinate $v_{i}$ 's as,

$$
\begin{gathered}
J 2=\min \left\{\bar{v}^{T}\left(Q_{1} / 4+\mu_{1} A_{c_{n+1}}+\mu_{2} A_{c_{n+2}}\right) \bar{v}\right. \\
\left.+\left(Q 2+\mu_{1} B_{c_{n+1}}+\mu_{2} B_{c_{n+2}}\right) \bar{v}\right\}
\end{gathered}
$$

which is a convex minimization problem and it is solved using matlab optimization toolbox. Convex optimization gives the $v_{i}^{\prime} s$ as real numbers, which are rounded off to the nearest number in $\{-1,1\}$, and this is the optimal result according to [7]. Finally, this result transformed back to the $u_{i}^{\prime} s$ to give the optimal control sequence.

\section{REsults AND Discussion}

A sample system response using the minimal energy On-Off optimal controller is given in the Fig. 4. In this first example, the a single leg link is driven to a desired final angle. When only a single leg link is to be controlled, the control input can be quite simple, as in the example shown. For comparison purposes, this On-Off controller was compared to the optimal linear-quadratic gaussian (LGQ) controller for the system, had feedback and analog rather than On-Off inputs been available. Both controllers produce qualitatively similar trajectories from the initial to the final value. In addition, the capacitative portion of the cost functions from the respective controllers is found to be less for the optimal On-Off controller, thanks to the minimal number of transitions that it dictates. The piezoelectric actuators used in the prototype system are formed from lead-zirconate-titanate and have very large resistance, such that over $99 \%$ of energy use is due to capacitive switching losses, as opposed to resistive holding losses.

To further explore the behavior of the prototype system using this On-Off control strategy, four situations are presented: 


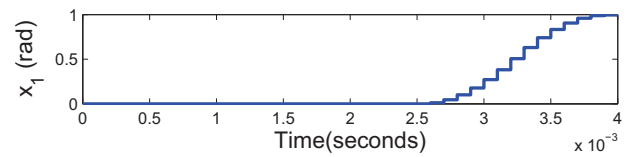

(a)

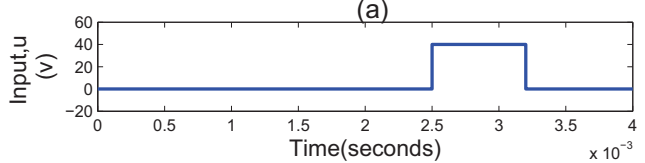

(b)

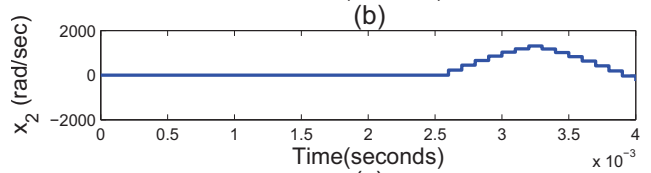

(c)

Fig. 4. Sample system output using optimal On-Off controller, showing (a) output angle $x_{1}$ (b) system input $u$ (c) output angular velocity $\dot{x}_{1}$

1) A comparison between the optimal On/Off controller found by this method and the optimal controller found via complete enumeration, i.e. a brute force optimization

2) Optimization results for a higher order system for various weights on positioning error

3) Variation with different relative weights for resistive and capacitative costs

4) Error due to parameter variation from the nominal model

\section{A. A comparison between the optimal On-Off controller and the brute force method}

To ensure that the optimization method is working properly, the result of the efficient optimization method was compared with that of brute force optimization over a shorter time period. In the brute force method all possible combinations of $u_{i}^{\prime} s$ were checked for feasibility and that with lowest cost, measured by the On-Off cost function, was selected. This approach is of course numerically inefficient, as one must check the cost for $2^{n}$ combinations of input, making it impractical for a larger a time period. However, is is possible to verify that for our sample system, the result of the brute force method matches the result obtained by the convex optimization method, which requires far fewer calculations.

\section{B. Optimization of a two mass system for various weights}

A full micro-robotic leg might consist of two links, driven by two separate actuators, in order to increase the total range of coverage by the leg, as shown in Fig. 5. The solution procedure described above can be easily augmented to optimize control of this system. The plots in Fig. 6 show the results obtained for the two mass system, under different weightings on the constraints. In the figure, $x_{3}$ and $x_{4}$ denote the angle and angular velocity of the $2^{\text {nd }}$ link relative to a stationary vertical line and the voltage applied to the two links at time step $k$ are denoted as $u_{1, k}$ and $u_{2, k}$, respectively.

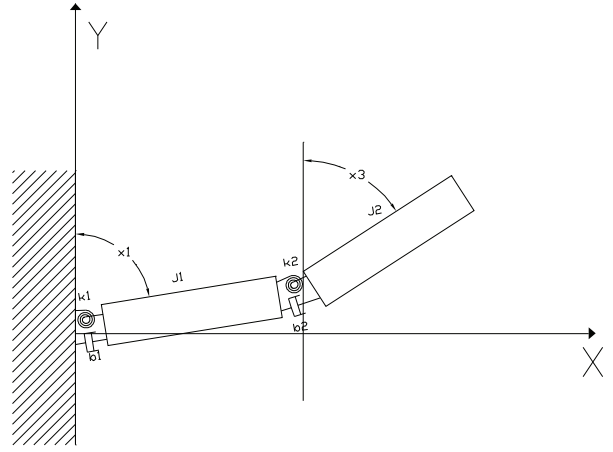

Fig. 5. Schematic view of a robotic leg with two links and two actuators, showing coordinate system for trajectory following

In each test case, the angles of the two links, states $x_{1}$ and $x_{3}$, are to be driven from a vertical position to final values of $0.5 \mathrm{rad}$ and $1 \mathrm{rad}$. Their angular velocities, states $x_{2}$ and $x_{4}$, are to return to values of zero at the conclusion of the time period. In the first set of plots, error in the final states is more heavily weighted, with $\mu_{1}=\mu_{2}=10^{7}$. As the penalty weighting is decreased, to $10^{5}$ in the following set of plots, a decrease in both accuracy and control effort (in the form of reduced 'on' time in this scenario) are seen, as is expected from the optimization algorithm.

\section{Variation with different weights on capacitative costs}

As the capacitance in the system is increased, the number of transitions decreases, as does the capacitative energy cost. Returning to the single leg link scenario, and examining a motion of longer duration, the effects of increasing capacitance in the system model are examined. Fig. 7(b) represents the lower-weighted capacitative cost (a $1 \mathrm{nF}$ capacitor) and the Fig. 7(c) corresponds to a more heavily weighted case (a $1 \mu \mathrm{F}$ capacitor). As seen from the Fig. 7(a) both of the responses reach the desired final position by the desired time, but the optimal On-Off input shows a larger number of transitions when capacitance is smaller. So from the results obtained, it can be concluded that the On-Off controller can be treated similar to a LQG controller in that it can be tuned to meet specific control requirements, placing more effort into minimizing switching cost, holding cost, or positioning error, as desired by the designer.

\section{Effects of parameter variation}

To explore robustness of the on-off controller, optimal on-off sequences were applied to plants with variation in stiffness, damping, and inertia parameter to as much as $15 \%$ of nominal values. The largest variation was seen for a $15 \%$ decrease in rotary inertia, $J$, as shown in Fig.8. The modeling error results in only a small error in the final states of the system. Further analysis of on-off controller robustness is a subject for future research, but these results indicate limited sensitivity of the algorithm to modeling error. 

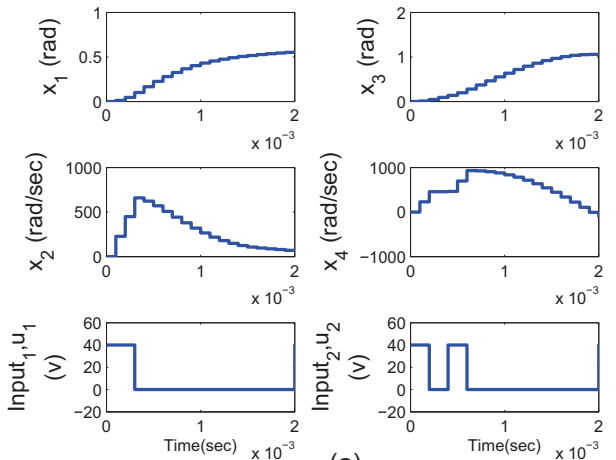

(a)
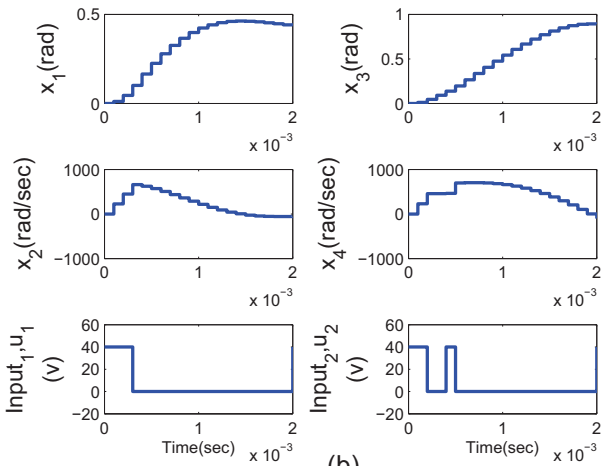

(b)

Fig. 6. States and control inputs with differing control weights for the two mass system (a) $\mu_{i}=10^{7}$ (b) $\mu_{i}=10^{5}$

\section{COnclusions And Future Work}

A method for generating an On-Off controller when switching costs are incorporated along with costs associated with 'on' time is presented. It is shown that switching costs can be added to existing On-Off controller design techniques while preserving the problem as one of convex optimization. Binary programming is used as an efficient technique for performing the convex optimization. In applications where only two input values are available to a controller, approaching this problem from the perspective of On-Off control can be a relatively simple method for identifying optimal On-Off control sequences, as compared to more general hybrid systems approaches that could alternatively be used to solve the problem. The behavior of the On-Off controller is explored for prototype problems involving one or two microrobotic leg joints driven by piezoelectric actuation, a situation where switching costs, in the form of capacitive energy consumption, are significant, but battery mass available to the system is severely constrained.

This approach to controller design is currently being expanded to accommodate other subsystems that might one day be available to an autonomous micro-robot. Charge recovery circuitry could reduce switching costs, while making additional voltage levels available for actuator control. Limited sensing capacity may also become available, which would provide the ability to alter the switching sequence from one step to another, or even in the process of a step.
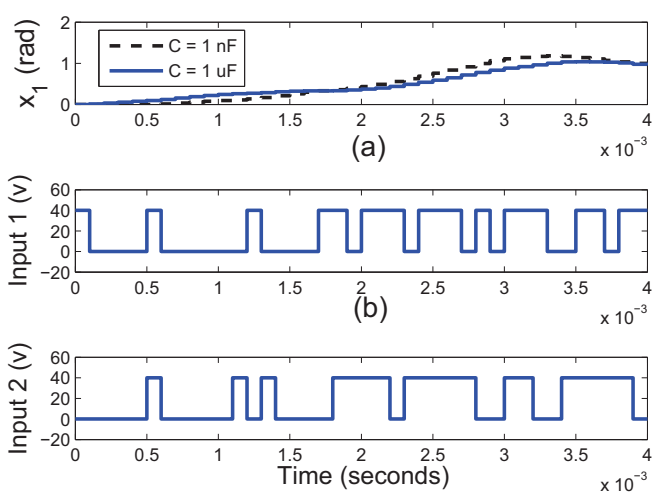

(c)

Fig. 7. Variation in input commands to a single link with different weights on capacitative costs (a) $\mathrm{C}=1 \mathrm{nF}$ (b) $\mathrm{C}=1 \mu \mathrm{F}$
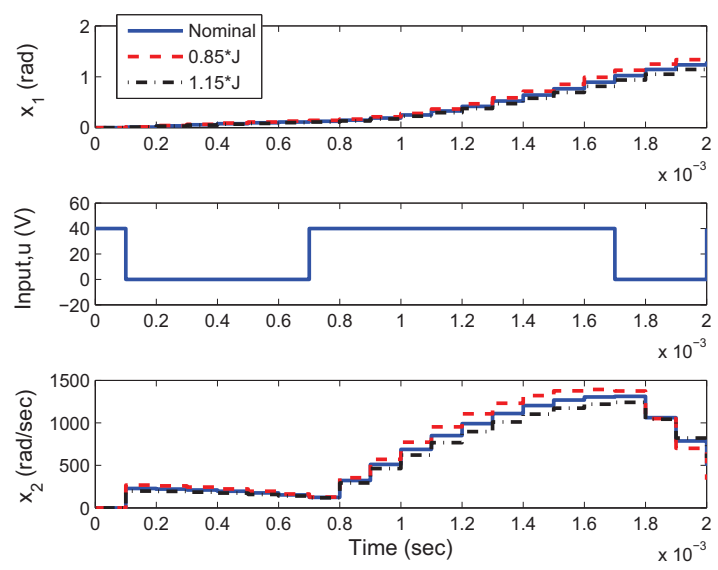

Fig. 8. Variation with Moment of inertia of the link

Ultimately, individual optimized robotic leg motions will be implemented sequentially to allow for efficient motion of the robot platform over a prescribed trajectory.

\section{REFERENCES}

[1] K. Oldham, B. Hahn, and P. Park, "On-off control for low power servo control in piezoelectric micro-robotics," accepted for presentation at the ASME Dynamic Systems and Control Conference, Ann Arbor, MI, 2008

[2] J.A. Main, "Efficient power amplifiers for piezoelectric applications." Smart Materials and Structures, vol. 5, 1996, pp. 766-775.

[3] Brian J. Driessen. "On-Off minimum-time control with limited fuel usage: near global optima via linear programming." In Proceedings of the American Control Conference,Chicago, Illinois June 2000.

[4] C.Y. Kaya and J.L. Noakes, "Computational method for time-optimal switching control", Journal of Optimization Theory and Applications, vol. 117 , no. 1,2003 , pp. 69-92.

[5] A. Bemporad and N. Giorgetti, "A sat-based hybrid solver for optimal control of hybrid systems," Hybrid Systems: Computation and Control: 7th International Workshop, 2004, pp 126-141.

[6] A. Bemporad, N. Giorgetti, "A logic-based hybrid solver for optimal control of hybrid systems," Proceedings of the IEEE Conference on Decision and Control, 2003, pp. 640645.

[7] D. Li and X. Sun, "Nonlinear integer programming," Springer, 2006. 